\title{
The Effect of Educational Intervention Based on the Transtheoretical Model on Stages of Change of Physical Activity in a Sample of Employees in Iran
}

\author{
Fatemeh Zare, Teamur Aghamolaei, ,"* $^{1}$ Mehdi Zare, ${ }^{3}$ and Amin Ghanbarnejad ${ }^{4}$ \\ ${ }_{1}^{1}$ MSc in Health Education, Health School, Hormozgan University of Medical Sciences, Bandar Abbas, IR Iran \\ ${ }^{2}$ Professor of Health Education, Social Determinants in Health Promotion Research Center, Department of Public Health, Faculty of Health, Hormozgan University of Medical \\ Sciences, Bandar Abbas, IR Iran \\ Sciences, Bandar Abbas, IR Iran

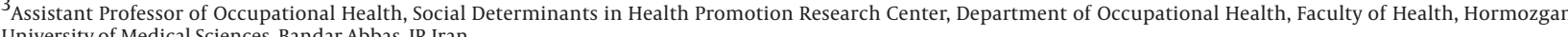 \\ University of Medical Sciences, Bandar Abbas, IR Iran \\ ${ }^{4}$ Instructor of Biostatistics, Social Determinants in Health Promotion Research Center, Department of Public Health, Faculty of Health, Hormozgan University of Medical Sciences, \\ Bandar Abbas, IR Iran \\ ${ }^{*}$ Corresponding author: Teamur Aghamolaei, Social Determinants in Health Promotion Research Center, Department of Public Health, Faculty of Health, Hormozgan University of \\ Medical Sciences, Bandar Abbas, IR Iran. Tel: +98-7613338583, Fax: +98-7613338584, E-mail: teaghamolaei@gmail.com
}

Received 2014 October 5; Revised 2015 June 10; Accepted 2015 October 28.

\begin{abstract}
Background: Promotion of physical activity is one of the most effective strategies for reducing the risk of certain chronic and noncommunicable diseases.

Objectives: To investigate the effect of an educational intervention, based on the transtheoretical model of stages of change of physical activity, on the employees of Abu Musa Island, in the south of Iran.

Materials and Methods: Different offices of Abu Musa Island including those for health, education, municipalities, civil, government, telecommunications, and welfare were divided randomly into intervention and control groups such that three offices were in intervention group and the rest were in control group. Forty employees were randomly selected from each group intervention and control and asked to complete survey questionnaires before, three, and six months after the educational intervention based on transtheoretical model. Data were analyzed by SPSS software version 19 . Sample t-test, repeated measure analysis and Wilcoxon tests were used to analyze the data.

Results: Stages of change of physical activity in the intervention group changed significantly three $(\mathrm{P}<0.01)$ and six months $(\mathrm{P}<0.04)$ after intervention in comparison with before intervention; however, there was no significant changes in stages of change of physical activity in control group. Three months after the intervention, perceived benefits and self-efficacy for exercise improved significantly $(\mathrm{P}<$ 0.05) in the intervention group. Further, the self-efficacy mean score six months after the intervention was significantly higher than preintervention $(\mathrm{P}<0.01)$. However, no change was observed in control group.

Conclusions: The results of this study showed that an educational intervention based on transtheoretical model can improve stages of change in physical activity behavior. It can also enhance cognitive factors such as perceived benefits and self-efficacy for exercise up to three months after the intervention, but over the time changes may decrease.
\end{abstract}

Keywords: Education, Employee, Physical Activity, Stages of Change, Transtheoretical Model

\section{Background}

An observable consequence of relying on machines and technology in 21st century is decreased physical activity (1). Sedentary lifestyle increases the risk of the major mortality causes and doubles the risk of cardiovascular diseases (2). Regular physical activity as a health-promoting behavior prevents or delays chronic diseases and mortality, reduces depression and anxiety, and improves mental health, life satisfaction, and quality of life (3). WHO recommends at least 30 minutes of physical activity per day, which is important for the prevention of cardiovascular diseases (2).

Despite the benefits of physical activity, in many countries, including Iran, a large number of individuals do not engage in regular physical activity (1). According to WHO statistics, more than 60 percent of the world's adults are physically inactive (4). A large proportion of workers in many countries do not have enough physical activity and naturally do not enjoy its benefits (4). The results of 27 cohort studies have shown that regular physical activity can reduce the risk of heart attacks from 35 to 55\%. These studies have also indicated that lack of activity is effective in causing cardiovascular diseases more than any other factor (smoking, cholesterol, and high blood pressure) (5). Studies in Iran have shown that over $70 \%$ of people do not receive enough physical activity (1). These facts reveal the need for implementation of interventions that promote public physical activity. Further, it seems that effective behavioral interventions are needed to ensure sustainable engagement in physical activity because half the people who begin physical activity stop it in less than six months (1).

Copyright (C) 2016, Health Promotion Research Center. This is an open-access article distributed under the terms of the Creative Commons Attribution-NonCommercial 4.0 International License (http://creativecommons.org/licenses/by-nc/4.0/) which permits copy and redistribute the material just in noncommercial usages, provided the original work is properly cited. 
Individuals need help with passing through different stages of physical activity behavior and promoting their physical activity. One of the most useful models in this regard is the transtheoretical model (4). The transtheoretical model has been widely identified as a framework for understanding health-related behaviors and conducting efforts to promote health (6). This model deals with behavior change and assumes that people pass five stages of behavior change. According to this model to achieve any type of sustainable change in behavior, people pass through the following stages: pre-contemplation (not thinking about changing the behavior), contemplation (thinking about changing behavior in the next six months), preparation (thinking about changing the behavior in the next month), action (having significant behavior change, but not as long as six months), and finally maintenance (to maintain the behavior for a period of six months or more) (7).

Studies have shown that the role of attitude, self-efficacy, and perceived benefits and barriers in changing people's behavior varies and depends on the stage of change (8). Perceived benefits and barriers related to regular physical activity are the positive and negative cognitive factors, respectively, which could encourage or discourage regular physical activity. In addition, studies have shown that individuals' self-efficacy is a significant factor in the promotion of physical activity behavior (3).

The application and effectiveness of the transtheoretical model in planning effective interventions toward the promotion of physical activity behavior have been investigated $(1,5,9,10)$. Studies have shown that interventions, based on the transtheoretical model, that have been adjusted to the stage of behavior change are faster and more effective than general interventions $(8,11)$. The transtheoretical model is unique because it emphasizes the stages of behavior change over specified time periods (12), while many other models focus only on the behavior. We used the transtheoretical model in this study to identify the educational needs of the participants according to the stages they are in, and to prepare them to progress to the next stage.

\section{Objectives}

The aim of this study was to investigate the effect of an educational intervention based on the transtheoretical model on the stages of change in physical activity behavior of the employees of Abu Musa Island, in the south of Iran.

\section{Materials and Methods}

\subsection{Participants}

Employees working in the offices of Abu Musa Island, in the south of Iran, were chosen as participants. First, different offices of Abu Musa Island including those dealing with health, education, municipality, civil, telecommunications, and welfare were randomly divided into control and intervention groups. Six offices included in the survey. four offices were in the control and three offices formed the intervention group. only three offices (health, education, municipality offices) included in the intervention group. Forty employees were randomly selected from the control group and 40 from the intervention group. The participants were matched in terms of age, sex, and education. The inclusion criteria were as follows: participants had to be residents of Abu Musa Island for at least six months from the time of the study, had to agree to participate actively in the study, did not have any conditions that inhibited physical activity.

\subsection{Ethical Considerations}

This study was approved by Research Ethics Committee of Hormozgan University of Medical Sciences and conducted in accordance with the Declaration of Helsinki (13). Written informed consent was obtained from all participants.

\subsection{Instruments}

We used a self-administered questionnaire consisting of four sections. The first section covered demographic information such as age and sex. The second section was related to the stages of change of physical activity. We used the physical activity staging (PAS) questionnaire for this (14). On the basis of their responses to questions from this section, they were classified into different physical activity stages: precontemplation, contemplation, preparation, action, and maintenance. It must be noted that physical activity in this study refers to any kind of exercise or physical activity that lasts for at least $30 \mathrm{~min}$ utes and is repeated at least 4 times a week and causes rapid heartbeat, breathing, or sweating. The reliability and validity of the PAS questionnaire has been confirmed in previous studies. The Cronbach's alpha coefficient of the responses in a pilot study have been reported to be 0.85 , which indicates an acceptable level of reliability of the questionnaire (15).

The third section of questionnaire pertained to perceived benefits of and perceived barriers to exercise. These were assessed by the exercise benefits/barriers scale (EBBS) (16). EBBS consists of a total of 43 items. Participants were asked to rate their agreement to perceived benefits and perceived barriers on a 4-point Likert scale (each item rated from strongly agree $=4$ to strongly disagree $=1$ ). The instrument can be scored and used in its entirety or as two separate scales (16). In this study it was scored on two separate scales. The overall perceived-benefits score was calculated by summing the scores of 29 benefit items, with higher values indicating greater perceived benefits. Scores on the benefits scale could range from 29 to 116. The overall perceived-barriers scores were calculated by summing the 14 barrier items, with higher values indicating greater perceived barriers. Scores on the barriers scale could range from 14 to 56 . Reliability 
and validity of this questionnaire had been confirmed by a previous study in Iran. The Cronbach's alpha coefficient for EBBS had been reported to be 0.87 (17).

The last section of the questionnaire was on self-efficacy. Self-efficacy was measured by an 8-item scale (3). Participants were asked to rate their confidence in their ability to do exercise on a 5-point Likert scale (from absolutely not true $=1$ to very true $=5$ ). Scores on the self-efficacy scale could range from 8 to 40 . A higher score indicated greater self-efficacy. Reliability and validity of this questionnaire had also been confirmed in previous study in Iran. The Cronbach's alpha coefficient for the self-efficacy questionnaire had been reported to be 0.83 (17). The questionnaires were completed before, three, and six months after the educational intervention.

\subsection{Intervention}

On the basis of the results from the questionnaires and the transtheoretical model of behavior change, participants were classified into 5 groups (precontemplation, contemplation, preparation, action and maintenance), and the educational needs of the groups were determined. Educational interventions for the participants was delivered according to the stages in which they were. Teaching methods included lectures, group discussions, educational videos, posters and educational pamphlets.

To provide more information to participants who were in the precontemplation and contemplation stages regarding the benefits of physical activity and the risks of sedentary lifestyle, two lecture sessions, each lasting an hour, were held. Because of the low number of individuals in the precontemplation stage, they were trained together with the contemplation group. The participants of maintenance group were also invited to attend the session on the risks of sedentary lifestyle, and they spoke about the impact of exercise on their lives. In addition, videos and pamphlets about the benefits of physical activity were distributed, and educational posters were posted in their offices.

In order to increase the awareness of those in the preparation stage, a lecture session lasting an hour was held to discuss the types of exercise, its benefits, and how to start exercising. The next session was a group discussion that prepared the individuals emotionally for change, by examining the barriers to exercise and finding solutions that targeted the individuals' affective domain. At this stage, given that individuals were ready to enter the action phase, participants were encouraged to engage in exercise and provided educational CDs. Educational pamphlets distributed among this group covered common perceived barriers to exercise for employees and strategies to overcome these barriers.

Because of the low number of individuals in the action stage, this group and maintenance group were pooled together. Members in these stages attended the educational sessions which conducted for precontemplation and contemplation groups and spoke about the benefits that they had gained through exercise. Then in a group discussion, a fitness instructor answered specific questions of participants who were in the preparation and maintenance stages. In this session, problems encountered during exercise and the strategies to overcome them were discussed. At the end of this session, participants were asked about their favorite sports for the purpose of creating educational CDs. A few days later, educational CDs and pamphlets specific to each group were distributed. The pamphlets for the action group included a proper regular exercise program and techniques to make exercise a fun activity. The pamphlets for the maintenance group included strategies to plan a regular exercise program and some educational material on the dos and don'ts of exercising.

\subsection{Statistical Analysis}

Data were analyzed by SPSS software version 19. Independent sample t-test, repeated measure analysis, and Wilcoxon tests were used.

\section{Results}

Demographic characteristics of the participants are presented in Tables 1 and 2. The mean age of participants in the intervention group was $33.9 \pm 7.7$ years (range $=21$ to 49 years), and the mean age of participants in the control group was $36.7 \pm 9.5$ years (range $=24$ to 56 years). Fifty percent of the participants in intervention group and $55 \%$ in the control group were male. There was no significant difference between intervention and control group in terms of age and sex $(P>0.05)$. Similarly, no significant difference was observed between the intervention and control groups in terms of the stages of change of behavior before the educational intervention $(P>0.05)$; however, the differences were significant three and six months after the educational intervention $(\mathrm{P}<0.05)$. Before the educational intervention, $20 \%$ of the participants in the intervention group were in the precontemplation stage and this number decreased to $5 \%$ three and six months after the intervention. Moreover, before the educational intervention, $10 \%$ of the participants in the intervention group were in the action stage, and this increased to 30\% three months after the educational intervention. Also, before the intervention $30 \%$ of the participants in the intervention group were in the maintenance stage, and this increased to $35 \%$ six months after the educational intervention (Table 3).

The results show that the stages of change of physical activity behavior in the intervention group improved significantly three months and six month after the intervention $(\mathrm{P}<0.01$ and $\mathrm{P}<0.04$, respectively) (Table 3$)$. However, no significant improvement in the stages of change of physical activity behavior were observed in the control group three and six months after intervention No intervention was administered to the control group. Educational intervention administered only to the intervention group. $(\mathrm{P}>0.05)$ (Table 4$)$. 
Zare F et al.

\begin{tabular}{lccc}
\hline \multicolumn{2}{l}{ Table 1. Comparison of Quantitative Demographic Variables in Intervention and Control Groups } \\
\hline Variable & Mean & Standard Deviation & P Value \\
\hline Age, $\mathbf{y}$ & & & 0.15 \\
$\quad$ Intervention & 33.9 & 9.5 & 7.7 \\
$\quad$ Control & 36.7 & & 0.13 \\
Job Experience, $\mathbf{y}$ & & 7.1 & 8.7 \\
$\quad$ Intervention & 9.9 & & \\
\hline Control & 12.7 & & \\
\hline
\end{tabular}

\begin{tabular}{lccc}
\hline \multicolumn{1}{l}{ Table 2. Comparison of Quantitative Demographic Variables in Intervention and Control Groups } & \\
\hline Variable & Intervention Group & Control Group & P Value \\
\hline Gender: Male & $20(50)$ & $28(70)$ & 0.06 \\
Female & $20(50)$ & $12(30)$ & 0.03 \\
Marital Status: Single & $13(32.5)$ & $4(10)$ & 0.13 \\
Married & $27(67.5)$ & $36(90)$ & $6(15)$ \\
Education: Under Diploma & $2(5)$ & $14(35)$ & 0.36 \\
Diploma & $10(25)$ & $20(50)$ & $4(10)$ \\
Higher Than Diploma & $28(70)$ & $36(90)$ & \\
\hline Smoking: Yes & $1(2.5)$ & & \\
\hline No & $39(97.5)$ & & \\
\hline
\end{tabular}

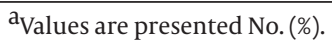

Table 3. Stages of Change in Physical Activity Behavior Before, Three, and Six Months After the Educational Intervention in the Intervention Group (Wilcoxon Test)

\begin{tabular}{lccc}
\hline Stages of Change & Before & After 3 Months & After 6 Months \\
\hline Precontemplation & $8(20)$ & $2(5)$ & $2(5)$ \\
Contemplation & $2(5)$ & $3(7.5)$ & $3(7.5)$ \\
Preparation & $14(35)$ & $11(27.5)$ & $14(35)$ \\
Action & $4(10)$ & $12(30)$ & $7(17.5)$ \\
Maintenance & $12(30)$ & $12(30)$ & $14(35)$ \\
PValue $^{\text {b }}$ & & 0.01 & 0.04 \\
\hline
\end{tabular}

${ }_{\text {Values are presented frequency (\%). }}$

${ }^{\mathrm{b}}$ Compared with before the educational intervention.

Table 4. Stages of Change in Physical Activity Before, Three, and Six Months After the Educational Intervention in the Control Group $(\text { Wilcoxon Test) })^{\mathrm{a}}$

\begin{tabular}{lccc}
\hline Stages of Change & Before & After 3 Months & After 6 Months \\
\hline Precontemplation & $8(20)$ & $7(17.5)$ & $7(17.5)$ \\
Contemplation & $5(12.5)$ & $9(22.5)$ & $4(10)$ \\
Preparation & $10(25)$ & $8(20)$ & $13(32.5)$ \\
Action & $6(15)$ & $5(12.5)$ & $4(10)$ \\
Maintenance & $11(27.5)$ & $11(27.5)$ & 0.52 \\
PValue $^{\text {b }}$ & & 0.44 & $(30)$ \\
\hline
\end{tabular}

${ }^{\mathrm{a}}$ Values are presented frequency (\%).

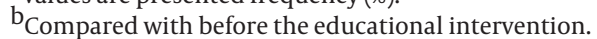


Zare F et al.

\begin{tabular}{|c|c|c|c|c|c|c|}
\hline \multirow[t]{2}{*}{ Variable } & \multicolumn{2}{|c|}{ Before Intervention } & \multicolumn{2}{|c|}{ Three Months After Intervention } & \multicolumn{2}{|c|}{ Six Months After Intervention } \\
\hline & Mean (SD) & PValue $^{\mathrm{a}}$ & Mean (SD) & P Value $^{\mathrm{a}}$ & Mean (SD) & P Value $^{\mathrm{a}}$ \\
\hline \multicolumn{7}{|c|}{ Perceived Benefits } \\
\hline Intervention & $97.4(15.6)$ & 0.9 & $103.5(10.5)$ & 0.04 & $102.7(9.9)$ & 0.10 \\
\hline Control & $97.8(14.2)$ & 0.9 & $98.5(12.7)$ & 0.04 & $98.4(13.1)$ & 0.10 \\
\hline \multicolumn{7}{|c|}{ Perceived Barriers } \\
\hline Intervention & $29.9(6.7)$ & 0.4 & $28.2(6.6)$ & 0.78 & $27.9(4.8)$ & 0.87 \\
\hline Control & $28.5(7.8)$ & 0.4 & $28.6(8.1)$ & 0.78 & $28.1(7.5)$ & 0.87 \\
\hline \multicolumn{7}{|l|}{ Self-Efficacy } \\
\hline Intervention & $23.2(6.9)$ & 0.6 & $26.9(5.9)$ & 0.01 & $27.7(6.6)$ & 0.03 \\
\hline Control & $24.0(8.0)$ & 0.6 & $23.5(6.3)$ & 0.01 & $24.5(5.7)$ & 0.03 \\
\hline
\end{tabular}

${ }^{\mathrm{a}}$ Independent sample t-test.

No significant differences were observed in the mean scores of perceived benefits, perceived barriers, and selfefficacy between the intervention and the control group before educational intervention. Three months after the educational intervention, the mean scores of perceived benefits and self-efficacy in the intervention group were significantly higher than those of the control group $(\mathrm{P}<$ 0.05 ), but there was no significant difference in the mean scores of perceived barriers between the intervention and control groups three months after the educational intervention $(P>0.05)$. Six months after the educational intervention, the mean score of self-efficacy in the intervention group was significantly higher than that in the control group $(P<0.03)$, but there was no significant difference in the mean scores of perceived benefits and perceived barriers between the intervention and control groups $(\mathrm{P}>0.05)$ (Table 5).

The mean scores of perceived benefits and self-efficacy differed significantly in the intervention group before, three, and six months after the intervention $(\mathrm{P}<$ 0.05); however, the differences in the mean scores of perceived barriers was not significant $(P>0.05)$. There was no significant difference in the mean scores of perceived benefits, perceived barriers, and self-efficacy in the control group before, three, and six months after intervention $(P>0.05)$. The results of the repeated measure analysis (Tables 6 and 7) show a significant difference in the mean scores of self-efficacy and perceived benefits before, three months, and six months after the intervention in the intervention group but not in the control group. For better illustration of changes in perceived benefits, perceived barriers, and self-efficacy during the time of intervention, three line plots for intervention group (intervention, control) are presented in Figures 1- 3.

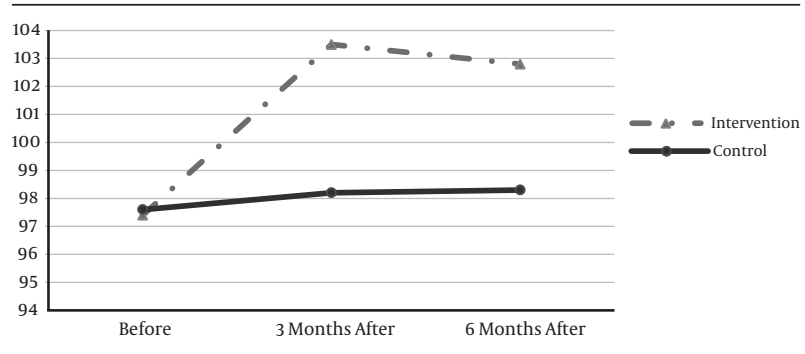

Figure 1. Changes in Perceived Benefits Before, Three, and Six Months After the Educational Intervention in the Intervention and Control Groups

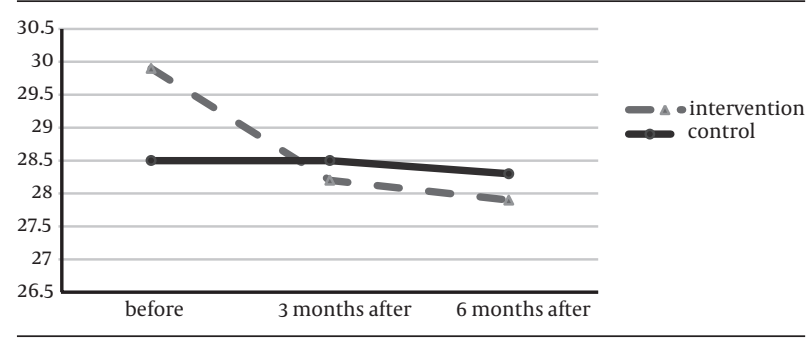

Figure 2. Changes in Perceived Barriers Before, Three, and Six Months After the Educational Intervention in the Intervention and Control Groups

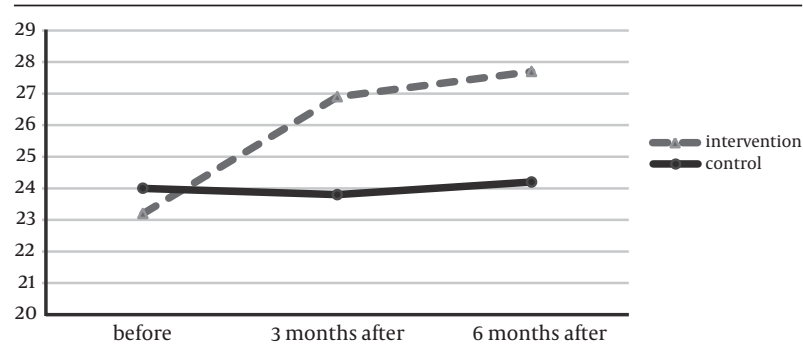

Figure 3. Changes in Self-Efficacy Before, Three, and Six Months After the Educational Intervention in the Intervention and Control Groups 
Zare F et al.

\begin{tabular}{|c|c|c|c|c|c|}
\hline Variables & Sum of Squares & Degree of Freedom & Mean Squares & F Value & P Value \\
\hline \multicolumn{6}{|l|}{ Perceived Benefits } \\
\hline Intervention Time & 10.7 & 1.2 & 8.8 & 0.12 & 0.77 \\
\hline Error & 2963.2 & 42.4 & 69.8 & 0.12 & 0.77 \\
\hline \multicolumn{6}{|l|}{ Perceived Barriers } \\
\hline Intervention Time & 754 & 1.1 & 0.67 & 0.01 & 0.97 \\
\hline Error & 1642.5 & 41.3 & 39.6 & 0.01 & 0.97 \\
\hline \multicolumn{6}{|l|}{ Self-Efficacy } \\
\hline Intervention Time & 2.5 & 1.4 & 1.7 & 0.09 & 0.58 \\
\hline Error & 1039.4 & 74 & 14 & 0.09 & 0.58 \\
\hline
\end{tabular}

\begin{tabular}{|c|c|c|c|c|c|}
\hline Ariables & Sum of Squares & Degree of Freedom & Mean Squares & F Value & P Value \\
\hline \multicolumn{6}{|l|}{ Perceived Benefits } \\
\hline Intervention Time & 825.2 & 1.4 & 559.3 & 6.2 & 0.008 \\
\hline Error & 4744.7 & 53.1 & 89.3 & 6.2 & 0.008 \\
\hline \multicolumn{6}{|l|}{ Perceived Barriers } \\
\hline Intervention Time & 94 & 2 & 47 & 2.28 & 0.1 \\
\hline Error & 1602.6 & 78 & 20.5 & 2.28 & 0.1 \\
\hline \multicolumn{6}{|l|}{ Self-Efficacy } \\
\hline Intervention Time & 457.5 & 2 & 228.7 & 11 & 0.001 \\
\hline Error & 1621.1 & 78 & 20.87 & 11 & 0.001 \\
\hline
\end{tabular}

\section{Discussion}

This study investigated the effectiveness of an educational intervention, based on the transtheoretical model, for increasing employees' physical activity. Stages of change in physical activity behavior in the intervention group, unlike the control group, improved significantly after three months of intervention. This result matches those of previous studies, which have reported that educational interventions based on the transtheoretical model can increase physical activity $(1,9,10$, 18-23). However, Hutchison et al. studied the interventions based on the transtheoretical model for changing physical activity behavior and showed that the model may not be the most appropriate for changing physical activity behavior. They argued that most of previous studies had not considered all the aspects of transtheoretical model and concluded that the effectiveness of this model cannot be judged until the interventions applied it properly (24).

Our comparison of the stages of behavior change three and six months after the intervention revealed that a number of individuals returned to past stages in the process of behavior change. For instance, some individuals in the action stage returned to the contemplation stage. This possibly because the individuals did not experience the expected benefits (such as weight loss) of physical activity, which could have led to a reduction in their per- ceived benefits of physical activity. This result highlights that to improve physical activity behavior, more attention should be paid to individuals who are in the action stage. Further, investigations should be carried out to identify the reasons for regressing from an advanced stage to a past one, to better design interventions.

In this study, the least number of return to a previous stage were seen among individuals in the maintenance stage. This is in agreement with the findings of Prochaska et al., who showed although individuals may return to an earlier stage at any point in the behavior change process, it is less likely among individuals in the maintenance stage (25). Therefore, to be successful, educational interventions should promote behavior that helps individuals attain the maintenance stage.

Results of the repeated measure analysis showed that in the intervention group the mean scores of perceived benefits of exercise were significantly different before and three and six month after the intervention (Tables 6 and 7). Three months after the intervention perceived benefits scores had significantly increased, which is in agreement with the results of some studies $(1,18,20)$. However, the mean score of perceived benefits declined slightly six months after the intervention, possibly because the participants may have forgotten the educational material 
over the time. This finding highlights the need for continuous education until individuals fully comprehend the benefits of exercise. Some of the common perceived benefits that employees cited in group discussions included a feeling of freshness, better sleep at night, less pain in the lower back, neck, and feet, fitness, and prevention of cardiovascular diseases. In a study on obese women, the perceived benefits of physical activity were reported to be a sense of achievement and success, increased energy, stress relief, and physical fitness after delivery (10).

As with the control group, in the intervention group, perceived barriers did not decrease after the intervention although other studies have shown that an educational intervention based on transtheoretical model can decrease the perceived barriers significantly $(1,18,20,21)$. The lack of decrease in perceived barriers can be attributed to the fact that some barriers could not be remedied by the educational intervention. For example barriers like remotely located exercise places and lack of encouragement by friends and family members cannot be removed through an educational intervention among the participants. Other solutions to reduce such barriers should be considered. Some solutions may include communication with relevant agencies such as the Ministry of Youth and Sports and the municipalities to increase public access to sporting facilities and installing sport equipment in all the parks. Further, barriers like "not having free time to exercise" could not be addressed through our educational intervention, and other solutions such as encouraging managers to allocate some place and time for employees' physical activity in the offices should be considered.

Individuals who were in the preparation stage and did not enter the action stage despite the educational intervention cited not having free time, being away from sport places, warm weather, and long working hours as the greatest barriers to physical activity. Similar factors have been identified by studies. In a study by Tavakoli et al., the main perceived barrier for physical activity was reported to be not having free time for physical activity (26). Fahrenwald and Walker found that some of the perceived barriers to exercising were getting tired after exercise, cold weather, and child care (10). Aghamolaei et al. showed that the barriers to exercise among students are shortage of time and feeling tired after the exercise. They also found that factors like self-efficacy and support from friends encourage students to engage in physical activity (3). One of barriers which for the older participants was that sport clubs lack specific arrangements for old people to exercise. This is an interesting insight, and we believe that governments should plan to develop sport teams and clubs for older people to encourage physical activity.

Some strategies proposed by participants of this study to reduce physical activity barriers included increasing access to sports facilities, training one of the employees to be a fitness trainer, holding sports classes at work, setting up a swimming pool and hiking group, and biking to work instead of using vehicles. According to Tavakoli et al., the most important measures to increase physical activity are facilitating access to exercise facilities, making exercise opportunities available in offices, and holding fitness classes at work (26).

Our results showed a significant difference in the mean scores of self-efficacy before and three and six months after the educational intervention in the intervention group while no such difference was observed in the control group (Tables 6 and 7). This finding is consistent with those of Moeini et al. (1), Hashemi et al. (18), Moradi et al. (20), and Dallow and Anderson (9). However, it is inconsistent with the results reported on Si et al. in their study on deaf youth (21).

A remarkable finding of this study is that unlike the scores of perceived benefits and stages of behavior change which decreased over the time, the self-efficacy score not only decreased but also increased slightly between three and six months after the intervention. This finding emphasizes that if self-efficacy can be increased through educational intervention, one's self-confidence for physical activity will probably improve, which can ensure longer compliance even if the educational intervention is not repeated. In addition, self-efficacy can indirectly boost physical activity by reducing the perceived barriers (18). Mazloomy et al. also showed that self-efficacy has an important role in increasing physical activity (27). Therefore, in educational interventions particular attention must be paid for strengthening this cognitive factor (28).

During the educational intervention, group discussions aiming to increase participants' awareness of physical activity benefits and the risks of inactivity were welcomed by participants, especially when the sessions were held during working hours. The posters on exercise at work were also greatly appreciated. Educational CDs (including instructions on swimming, aerobics, women's bodybuilding, fitness using balls, skating, and yoga) did not get much attention from individuals who were in the preparation, action, and maintenance stages. A notable point about the educational CDs was that individuals in the precontemplation and contemplation stages had not viewed the CDs even several weeks after they received them. This indicates that educational materials aimed at helping people learn on their own are not appropriate for those who are in precontemplation and contemplation stages. To ensure that individuals benefit from educational CDs and videos, the content should be aired during sessions and classes.

The results of this study show that educational interventions based on the transtheoretical model can improve stages of change of physical activity behavior and cognitive factors such as perceived benefits and self-efficacy for exercise up to three months after the intervention, but over the time (six months), the changes may decrease. This learnings from this study can be used for improvement of physical activity behavior. Efforts should be made to continue with the educational intervention until all the participants enter the maintenance stage. 


\section{Acknowledgments}

The authors wish to thank the participants for contributing to the research and responding to the questionnaire patiently.

\section{Footnotes}

Authors' Contribution:Fatemeh Zare collected the data and conducted the intervention. Teamur Aghamolaei was responsible for design and conducting the research and participated in preparing and editing the manuscript, as the corresponding author. Mehdi Zare wrote the draft of the manuscript. Amin Ghanbarnejad conducted the statistical analysis and prepared the results. All authors have read and approved the manuscript.

Funding/Support:This study was financially supported by the Research \& Technology department of Hormozgan University of Medical Sciences, Bandar Abbas, Iran.

\section{References}

1. Moeini B, Rahimi M, Hazaveie SM, Allahverdi Pour H, Moghim Beigi A, Mohammadfam I. Effect of education based on trans-theoretical model on promoting physical activity and increasing physical work capacity. J MIL Med. 2010;12(3):123-30.

2. Sharifirad G, Mohebbi S, Matlabi M. The relationship of physical activity in middle age and cardiovascular problems in old age in retired people in Isfahan. Q Horizon Med Sci. 2007;13(2):57-63.

3. Shen B, Xu C. Effects of self-efficacy, body mass, and cardiorespiratory fitness on exercise motives in Chinese college students. J Phys Act Health. 2008;5(5):706-18. [PubMed:18820345]

4. Prochaska JO, DiClemente CC. Stages and processes of selfchange of smoking: toward an integrative model of change. Consult Clin Psychol.1983;51(3):390-5. [PubMed: 6863699]

5. Plotnikoff RC, Blanchard C, Hotz SB, Rhodes R. Validation of the decisional balance scales in the exercise domain from the transtheoretical model: A longitudinal test. Measurement PHYS EDUC and Exercise Sci. 2001;5(4):191-206.

6. Aghamolaei T, Tvafian S, Zare S. Application of transtheoretical model for assessing stages of smoking behavior change in the adult population of Bandarabbas. Payesh. 2010;8(3):263-9.

7. Safari M, Shojaiizade D, Ghofranipoor F, Heidarnia A, Pakpoor Hajiagha A. Theories, models and methods of health education and health promotion [in Persian]. First ed. Tehran: Sobhan; 2010.

8. De Bourdeaudhuij I, Philippaerts R, Crombez G, Matton L, Wijndaele K, Balduck AL, et al. Stages of change for physical activity in a community sample of adolescents. Health Educ Res 2005;20(3):357-66. doi: 10.1093/her/cyg131. [PubMed: 15536126]

9. Dallow CB, Anderson J. Using self-efficacy and a transtheoretical model to develop a physical activity intervention for obese women. Am J Health Promot. 2003;17(6):373-81. [PubMed: 12858617]

10. Fahrenwald NL, Walker SN. Application of the Transtheoretical Model of behavior change to the physical activity behavior of WIC mothers. Public Health Nurs. 2003;20(4):307-17. [PubMed: 12823791]

11. Kim CJ, Kim BT, Chae SM. Application of the transtheoretical model: exercise behavior in Korean adults with metabolic syndrome. J Cardiovasc Nurs. 2010;25(4):323-31. doi: 10.1097| ICN.ob013e3181c8a3e8. [PubMed:20539166]

12. Jourdan PJ, Nigg CR, Norman GJ, Rossi JS. Integration attitude with decisional balance as predictors of stage of change for exercise. Psycho of Sport and Exercise. 2008;3:65-85.

13. Declaration of Helsinki - Ethical Principles for Medical Research Involving Human Subjects. World Medical Association; October 2013. Available from: http://www.wma.net/ en/30publications/10policies/b3/.

14. Nigg C, Hellsten L, Norman G, Braun L, Breger R, Burbank P, et al. Physical activity staging distribution: establishing a heuristic using multiple studies. Ann Behav Med. 2005;29 Suppl:35-45. doi 10.1207/s15324796abm2902s_7. [PubMed:15921488]

15. Aghamollaie T, Tvafian S, Zare S. Stages of physical activity behavior change in the population over 15 years, residents of Bandar Abbas [in Persian]. J payesh. 7(2):253-8.

16. Sechrist KR, Walker SN, Pender NJ. Development and psychomet ric evaluation of the exercise benefits/barriers scale. Res Nurs Health. 1987;10(6):357-65. [PubMed:3423307]

17. Aghamollaee T, Tvafian S, Hasani L. Self efficacy, perceived benefits and barriers to physical activity in students of Hormozgan university's medical sience [in Persian]. J Iran epidmiology 1387;4(3, 4):9-15.

18. Hashemi SZ, Rakhshani F, Navidian A, Mosavi SR. Effectiveness of Educational Program based on Trans-Theoretical Model on Rate of Physical Activity among Household Women in Zahedan, Iran. HEALTH SYSTEM RESEARCH. 2013;9(2):144-52.

19. Kirk AF, Higgins LA, Hughes AR, Fisher BM, Mutrie N, Hillis S, et al. A randomized, controlled trial to study the effect of exercise consultation on the promotion of physical activity in people with Type 2 diabetes: a pilot study. Diabet Med. 2001;18(11):877-82. [PubMed: 11703431

20. Hamule MM, Vahed AS, Eshgh ZM. Assessment of the Effect of Educational Program Based on Trans Theoretical Model (TTM) on Physical Activity in Patients with Inflammatory Bowel Diseases. Sci J of Hamadan U Med Sci. 2010;17(1):39-45.

21. Si Q, Yu K, Cardinal BJ, Lee H, Yan Z, Loprinzi PD, et al. Promot ing exercise behavior among Chinese youth with hearing loss: a randomized controlled trial based on the transtheoretica model. Psychol Rep. 2011;109(3):896-906. doi: 10.2466/06.11.13.15. PR0.109.6.896-906. [PubMed: 22420119]

22. Skaal L, Pengpid S. The predictive validity and effects of using the transtheoretical model to increase the physical activity of healthcare workers in a public hospital in South Africa Transl Behav Med. 2012;2(4):384-91. doi: 10.1007/s13142-012-0136-5. [PubMed: 23293685]

23. Woods C, Mutrie N, Scott M. Physical activity intervention a transtheoretical model-based intervention designed to help sedentary young adults become active. Health Educ Res. 2002;17(4):451-60. [PubMed:12197590]

24. Hutchison AJ, Breckon JD, Johnston LH. Physical activity behavior change interventions based on the transtheoretical model a systematic review. Health Educ Behav. 2009;36(5):829-45. doi: 10.1177/1090198108318491. [PubMed:18607007]

25. Prochaska JO. Transtheoretical model of behavior change. En cyclopedia of behavioral medicine. New York: Springer; 2013. pp. 1997-2000.

26. Tavakoli R, Sanaienasab H, Karimi A, Noparast M. Physical activity and factors influencing it among the personnel of a military center in Iran. J Res Health. 2012;2(2):172-80.

27. Mazloomy S, Mohammadi M, MorovatiSharifabad MA, Falahzade H. A study of the decisional balance's role in exercise status among yazd's staff on the basis process of change model. Zahedan Journal of Research in Medical Sciences. 2009;11(2).

28. Soleimani Ahmadi M, Vatandoost H, Shaeghi M, Raeisi A, Abed F, Eshraghian M R, Aghamolaei T, Madani A H, Safari R, Jamshid $\mathrm{M}$, Alimorad A, inventors. Effects of Educational Intervention on Long-Lasting Insecticidal Nets Use in a Malarious Area, Southeast Iran. Acta Medica Iranica. 2012;50(4):279-86. [PubMed: 22592579] 